\title{
Superconducting NbN detector for neutral nanoparticles
}

\author{
Markus Marksteiner, ${ }^{1}$ Alexander Divochiy, ${ }^{2}$ Michele Sclafani, ${ }^{1}$ Philipp Haslinger, ${ }^{1}$ Hendrik \\ Ulbricht, ${ }^{3}$ Alexander Korneev, ${ }^{2}$ Alexander Semenov, ${ }^{2}$ Gregory Gol'tsman, ${ }^{2}$ and Markus Arndt, * \\ ${ }^{1}$ Faculty of Physics, University of Vienna, Boltzmanngasse 5, A-1090 Vienna, Austria \\ ${ }^{2}$ Department of Physics, Moscow State Pedagogical University, \\ M. Pirogovskaya Street 1, Moscow 119992, Russia \\ ${ }^{3}$ School of Physics and Astronomy, University of Southampton, \\ Highfield, Southampton, SO171BJ, United Kingdom
}

(Dated: September 18, 2018)

\begin{abstract}
We present a proof-of-principle study of superconducting single photon detectors (SSPD) for the detection of individual neutral molecules/nanoparticles at low energies. The new detector is applied to characterize a laser desorption source for biomolecules and it allows to retrieve the arrival time distribution of a pulsed molecular beam containing the amino acid tryptophan, the polypeptide gramicidin as well as insulin, myoglobin and hemoglobin. We discuss the experimental evidence that the detector is actually sensitive to isolated neutral particles.
\end{abstract}

\section{INTRODUCTION}

The detection of isolated neutral molecules and nanoparticles in the gas phase is both a necessity and a challenge for many experiments that range from physical chemistry over environmental monitoring [1] to the foundations of physics. Our own work was originally motivated by matter wave interferometry with massive molecules 2-4] and applications in molecule metrology [5, [6]. The extension of such experiments to higher masses requires also improved methods for detecting neutral nanoparticles. While ionization techniques are routinely used for particles up to about $2000 \mathrm{Da}$, postionization of organic molecules beyond that mass has remained a significant challenge [7, 8]. Recent experiments observed photoionization of tryptophan-metal complexes and nucleotide clusters up to $6000 \mathrm{Da}$ [9, 10]. But for the majority of high-mass biomolecules this method seems to be precluded.

Hyperthermal surface ionization [12] was shown to allow the detection of some neutral molecules, with insulin currently setting the mass record [13].

Modern nanofabrication technologies also allow to build nanoscale oscillators which change their resonance frequency when their mass is augmented by even a single molecule. Such cantilever based detectors [14, 15] have rather a lower than an upper mass limit. They currently reach a sensitivity of below $200 \mathrm{Da}$ [16]. First proof-of principle mass spectrometer applications achieved the capability to detect single proteins [17].

Whenever mass cannot be measured directly, bolometer detectors 18 20] may convert molecular energy first into sensor temperature and then into an electrical signal. However, the translational energy of a single amino acid, such as tryptophan, does not exceed $0.3 \mathrm{eV}$, even at a molecular velocity of $500 \mathrm{~m} / \mathrm{s}$. This is why the first bolometers [21] still operated with a minimum detection

\footnotetext{
* markus.arndt@univie.ac.at http://www.quantumnano.at
}

threshold of about $10^{7}$ molecules per second. Superconductors were suggested as promising sensors [19, 22, 23] since their conductivity changes strongly with temperature in the vicinity of the phase transition edge.

The implementation of superconducting tunneling junctions (STJ) made it possible to detect charged individual molecules [24 26]. This is interesting for mass spectrometry because the STJ response depends on the particle's energy [27]. This allows to combine the mass discrimination of a time-of-flight spectrometer with a detector whose efficiency remains constant over a wide mass range. Tunneling junction detectors require, however, cooling well below $4 \mathrm{~K}$ and up to now they were only used for recording either ensembles of slow neutral particles or for detecting individual but energetic charged particles.

In this letter we present our first experimental evidence that a combination of both is feasible, i.e. a detector for single neutral molecules of low kinetic and low internal energy. Our nanowire detector was originally fabricated as a superconducting single photon detector. Its sensitivity to single photons was demonstrated across the entire spectrum from UV to mid-IR, with quantum efficiencies up to $30 \%$ 28 32]. Before we started the experiments, it was far from obvious that such a device would also be sensitive to slow nanoparticles. In the following we discuss the acquired evidence that this detector is capable of recording the incidence of isolated neutral biomolecules.

\section{EXPERIMENTAL SETUP}

The entire experiment consists of a pulsed molecular beam, a free flight trajectory in high vacuum and the superconducting nanowire detector in a differentially pumped helium cryostat, about $76 \mathrm{~cm}$ behind the source. 


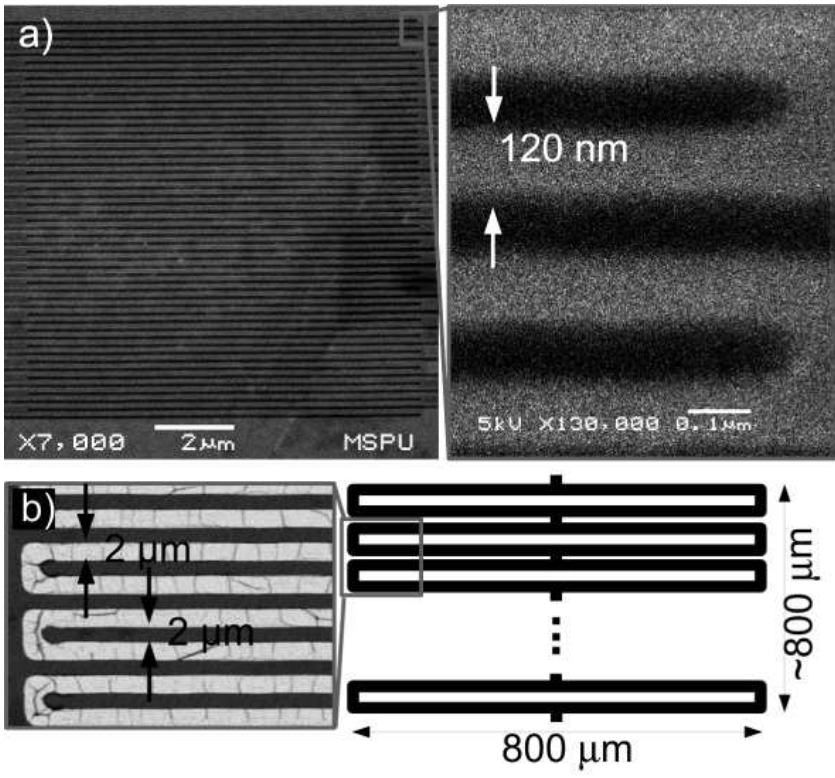

FIG. 1. (a) Electron microscopy image of the $10 \mu \mathrm{m} \times 10 \mu \mathrm{m}$ sensitive SSPD element. The NbN film appears is colored in grey. (b) Sketch (right) and SEM image (left) of the superconducting $\mathrm{NbN}$ bolometer. The bolometer strips and gaps are about $2 \mu \mathrm{m}$ wide.

\section{A. Superconducting detectors}

Two different types of detectors were tested: Superconducting single photon counting devices (SSPDs) and superconducting bolometers (SBs).

The SSPDs were fabricated by depositing a NbN film of $3.5 . .4 \mathrm{~nm}$ thickness on a sapphire substrate. We tested chips with an open area of either $10 \times 10 \mu \mathrm{m}^{2}$ or $20 \times 20 \mu \mathrm{m}^{2}$. The $100 . .120 \mathrm{~nm}$ wide superconducting wire meanders on the surface with a filling factor of $60 \%$. The critical temperature of $\mathrm{NbN}$ is $\mathrm{T}_{c}=10 . .11 \mathrm{~K}$ and the critical current density amounts to $j_{c}=3 . .5 \times 10^{6} \mathrm{~A} / \mathrm{cm}^{2}$.

The SSPD fabrication process was described in detail in [33]. In brief, $\mathrm{NbN}$ superconducting films were deposited on R-cut sapphire substrates by DC reactive magnetron sputtering in an $\mathrm{Ar}$ and $\mathrm{N}_{2}$ mixture. The film was patterned by direct electron beam lithography and reactive ion etching. Gold contacts were added using photolithography and wet etching. In Figure 1 we show electron microscopy images of the sensitive element. We operate the device in a liquid helium bath cryostat at $4.2 \mathrm{~K}$ and apply a DC bias current slightly smaller than the critical current. The signal is capacitively coupled from the chip to the oscilloscope (see Figure2).

The detection mechanism may be understood as follows: When a molecule hits the film surface, it creates high-energy acoustic phonons. These phonons are rapidly absorbed by the electron subsystem of the film due to their short inelastic mean free path. Excess quasiparticles are then created which, in turn, dispose of their energy by the emission of second generation phonons.
This triggers an avalanche multiplication cascade. The process is similar to what happens during photon detection. The distinctive difference lies in the first step: the photon detection cascade starts from a single high-energy quasiparticle created by the photon. The dynamics of the subsequent stages is determined only by the absorbed energy.

When the energy of the quasiparticles decreases to a value around $10 \mathrm{~K}$, the electron-electron interaction becomes more efficient for the multiplication of the quasiparticles than the electron-phonon interaction. Due to this fact, the main part of the energy, that is initially deposited in the film remains in the quasiparticle subsystem. At the end of the cascade, a hot spot of excess quasiparticles is formed and the supercurrent is forced to flow around the new normal-conducting area. If the hot spot is sufficiently large the current density in the 'sidewalks' increases beyond the critical current density. This results in a short breakdown of superconductivity across the entire width of the nanostripe and in a voltage pulse that can be easily detected 28 31]. For photons, a typical pulse response lasts over $10 \mathrm{~ns}$.

The second detector type that we tested was a classical superconducting bolometer. These chips were made from the same $\mathrm{NbN}$ film, again using photolithography and wet etching. Figure 1(b) presents the sketch of its sensitive element and an SEM image. The bolometer chip is working at the critical temperature $T_{c}$. The additional energy that is delivered to the surface by the impacting molecules may heat the superconductor above $T_{c}$ and cause a voltage peak. The incident energy has to sufficiently high to induce the required temperature change. Because of the large width of the stripes this condition can often not be met by a single molecule alone and the sensors responds only to many simultaneously impacting particles. With this second detector type we were not able to detect any molecular signal in our experiments. This type of superconducting wide-area bolometer is therefore not further discussed in the following where we rather focus on our explorations of nanostructured SSPD chips.

\section{B. Molecular beam source}

The details of our laser desorption source have already been described elsewhere [9, 10]: Organic molecules were laser desorbed by a Nd:YAG laser beam $(355 \mathrm{~nm}, 5 \mathrm{~ns}$, $6 . .10 \mathrm{~mJ}$ ), which was focused to a spot on a pressed powder sample (Figure2). The desorbed molecules are cooled and entrained by a jet of helium gas that fills the mixing channel before it exits through a $1 \mathrm{~mm}$ opening into a vacuum of $10^{-3}$ mbar. About $2 \mathrm{~cm}$ behind the mixing channel the beam passes a skimmer of $1 \mathrm{~mm}$ diameter, which separates the source from a differential pumping stage. In this second chamber the molecular beam is filtered by a copper mesh with $7.5 \mu \mathrm{m}$ openings. This microstructure is used to reject grains of powder that might 


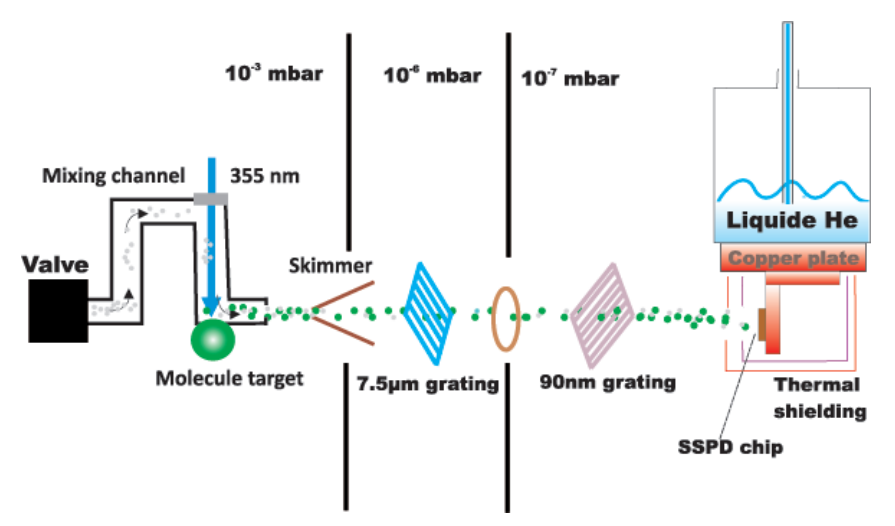

FIG. 2. A pulsed laser desorbs biomolecules, which are then entrained by a supersonically expanding noble gas jet. After two-fold mechanical filtering the molecular beam hits the superconducting NbN detector. The impact of molecules releases a voltage pulse which is stored by an oscilloscope and a timer card.

be ejected during the ablation process.

The stream of single molecules as well as possibly a background of microscopic particles leaves the second pumping stage through a $1 \mathrm{~cm}$ diameter opening into the detection chamber where the SSPD chip is attached to a Helium bath cryostat. The overall distance from the desorption spot to the superconducting chip is $76 \mathrm{~cm}$. For some experiments we added a second mechanical filter: a $\mathrm{SiN}_{x}$ line grating with a period of $266 \mathrm{~nm}$ and openings as small as $90 \mathrm{~nm}$ was attached to the entrance window of the cryostat. This addition further limited the transmitted particle size and also helped extending the life time of the SSPD chips, which was otherwise strongly affected by the accumulation of molecular material. Even with the additional filter in place, the active time of an individual chip was limited to about 20.000 desorption shots, time after which a layer of molecules had covered the surface and made it insensitive. It is known [11] that SSPD chips can even be used to resolve the energy of incident photons. Similar energy dependent measurements with molecules were, however, still impeded in our present proof-of-principle study by the time-varying surface coverage.

\section{RESULTS}

In order to explore its detection capabilities the SSPD chip was placed into the laser desorbed biomolecular beam. The first experiments were performed with a mixture of several molecules. It contained $0.2 \mathrm{~g}$ myoglobin $(17 \mathrm{kDa}), 0.3 \mathrm{~g} \beta$-carotene $(537 \mathrm{Da}), 0.3 \mathrm{~g}$ insulin $(5.8 \mathrm{kDa}), 0.25 \mathrm{~g}$ bovine serum albumin (BSA, $66 \mathrm{kDa})$, and $0.5 \mathrm{~g}$ cellulose of unspecified chain length to mechanically bind the other components.

In this first test we used a $20 \times 20 \mu \mathrm{m}^{2}$ SSPD chip and in Figure 3 we show a typical individual detection event from the desorbed molecule mixture. The peak is about $10 \mathrm{~ns}$ wide (FWHM) and indicates a high temporal detector resolution also for neutral nanoparticles.
We performed several tests to corroborate the evidence for the SSPD's sensitivity to isolated molecules and to exclude possible other reasons for the observed signals such as for instance the co-propagating seed gas or codesorbed cellulose:

The influence of the rapidly expanding seed gas can be tested by switching off the desorbing laser beam. We searched for signs of the expanding helium carrier gas pulse alone and the complete absence of any signal in this setting indicates that the SSPD chip is not capable of detecting individual helium atoms. The same is also true for all of the heavier noble gases such as neon, argon, krypton or xenon. None of them showed any detectable signal under our experimental conditions.

One might speculate that the higher kinetic energy of the more massive biomolecules could be outweighed by the larger number of lighter noble gas atoms. This argument would be supported by the fact that seed gas atoms must be much more abundant than the laser implanted biomolecules - otherwise supersonic expansion would never occur. However, since no signal was detected for the pure noble gas beams alone, a collective effect by the dense gas jet can be excluded. Since the biomolecular beam is more dilute than the carrier gas, a collective effect of organic particles is even less likely.

This finding is in variance to that for classic bolometers where the incidence of many particles was actually required to trigger a signal [21]. It has, however, to be noted that these detectors were not nanostructured and they were exploiting a different mechanism. Our result gives thus first evidence that the SSPD chip is indeed not sensitive to the intense particle flux of atoms but rather to the local energy density of single complex nanoparticles.

As mentioned before, the molecules were always admixed with cellulose to achieve mechanical stabilization of the sample. In order to separate matrix signals from analyte signals we also performed a separate desorption experiment with pure cellulose powder alone. The complete absence of any measurable signal indicates again 


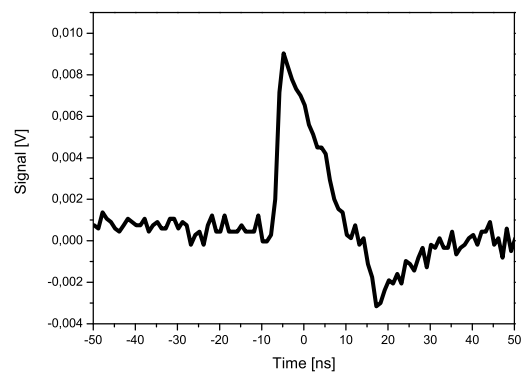

FIG. 3. A typical individual peak, that is attributed to the impact of neutral molecules on the chip. The $20 \times 20 \mu \mathrm{m}$ SSPD chip was biased with a current of $19.5 \mu \mathrm{A}$. The signal was amplified by $20 \mathrm{~dB}$.

that the ablated matrix particles do not contribute any background in the SSPD counter. In order to enable a more quantitative evaluation we switched to a pulse counting mode and recorded time-of-flight curves for various experimental settings. The molecular velocity may differ from that of a free supersonic expansion, since the molecules can be delayed inside the gas mixing channel before they exit. This delay may lead to an underestimation of the actual velocity. The velocities and kinetic energies below are therefore reasonable lower limits.

In all the following experiments the samples contained only biomolecules from one species mixed 1:1 with cellulose. In Figure 4 we show the arrival time distribution for a tryptophan (204 Da) and gramicidin (1.9 kDa) sample, respectively. Figure $4 \mathrm{~b}$ depicts the distributions for insulin, myoglobin and porcine hemoglobin $(66 \mathrm{kDa})$ sample. All curves in Figure 4 were recorded using the same $20 \times 20 \mu m$ SSPD chip, the same discriminator level, a bias current of $20 \pm 1 \mu \mathrm{A}$ and two particle filters in the beam line, i.e. a $7.5 \mu \mathrm{m}$ mesh as well as the $90 \mathrm{~nm} \mathrm{SiN}$ filter. The opening time of the valve was set to $700 \mu \mathrm{s}$ for all recorded curves in Figure 4 , except for the one of tryptophan as discussed below. The arrival time distributions in Figure 4 show a double structure which is a result of the particular valve setting in these experiments.

The agreement of the detected arrival times with the expected flight times may already be interpreted as a good indication for the detection of neutral particles. It is, however, desirable to corroborate this statement by complementary measurements which must rely on alternative detectors. As they are not readily available in the mass range beyond $2000 \mathrm{Da}$, where ionization detectors start to fail [7, 8] and nanomechanical detectors are not yet commercially available, it is also still open whether high mass molecules (e.g. hemoglobin) can survive the desorption process as intact particles or whether the observed signals are rather caused by smaller neutral fragments generated in the source.

This is why our first checks were focused on characterizing the molecular beam source for tryptophan and gramicidin, where it is known [34, 35] that VUV laser ionization is soft and capable of detecting isolated molecules
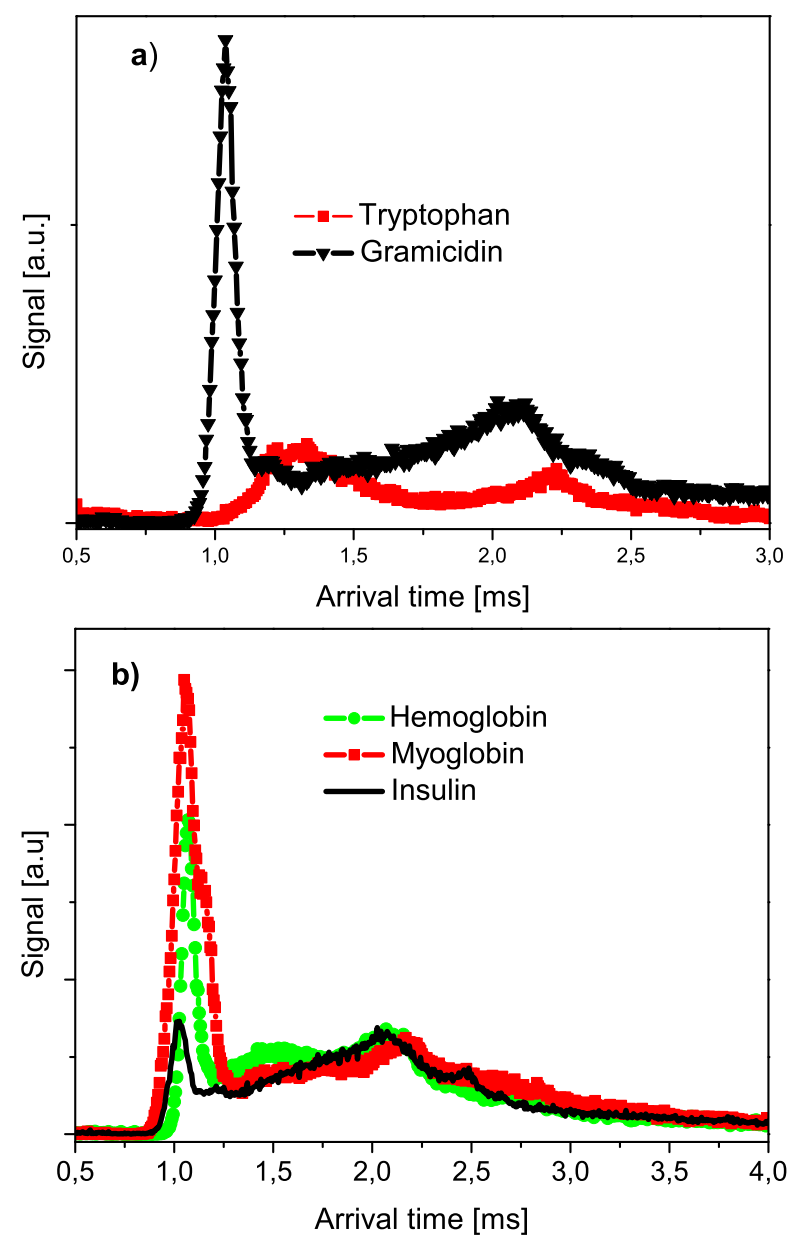

FIG. 4. A) Arrival time distribution of tryptophan and gramicidin. B) arrival time distribution of myoglobin, insulin and hemoglobin. All molecules were detected using the $20 \times 20 \mu \mathrm{m}$ SSPD chip.

as well as larger clusters 9,10$]$. A $\mathrm{F}_{2}$ laser $(157 \mathrm{~nm}$, $5 \mathrm{~ns}$, up to $3 \mathrm{~mJ}$ ) is here combined with time-of-flight mass spectrometry (TOF-MS) to reveal the arrival time and mass distribution of all molecules emerging from the source.

Figure[5 shows a representative arrival time distribution for tryptophan, measured using TOF-MS and the same source settings as in the SSPD experiments. Only the flight distance between source and detector was shortened to $0.5 \mathrm{~m}$. This reduces also the molecular flight times.

The arrival time distribution, recorded in photoionization TOF-MS at the mass of the single monomer (Figure (4) has the same structure as the signals recorded by the SSPD (Figure5). Small differences in the arrival times can be assigned to an accidental reduction of the valve opening time which influences both the pressure inside the mixing channel and the velocity of the expanding molecules. This is supported by Figure 6 which depicts the arrival time distribution for gramicidin for three different valve opening times recorded by the SSPD chip. 


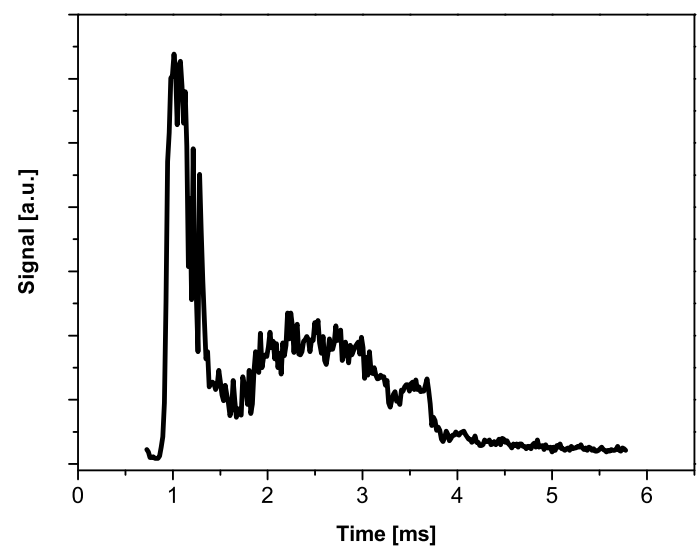

FIG. 5. Arrival time distribution of tryptophan recorded via laser postionization and time-of-flight (TOF) mass spectrometry. The shape of the TOF-distribution reproduces the distribution recorded by the superconducting chip (see figure 4).

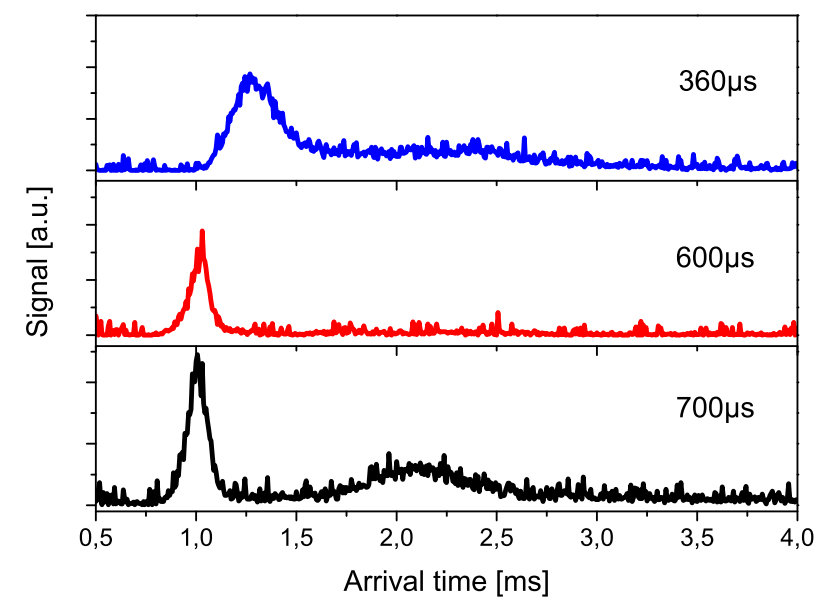

FIG. 6. Arrival time distribution of gramicidin for different valve opening times, using a $10 \times 10 \mu \mathrm{m}$ SSPD chip. A reduction of the valve opening time leads to shifted arrival time distributions (slower molecules).

For short pulse times (Figure6, top) the arrival time distribution resembles the one recorded for tryptophan in 4a, where the molecule arrival time was delayed. Interestingly, valve times around $600 \mu \mathrm{s}$ do not show the double structure, as can be seen in Figure6, center. We chose a slightly higher opening time of $700 \mu$ s (Figure6, bottom) for our experiments. This causes a double peak in the arrival time but also adds to the signal.

From these tests we gather the general insight that the arrival time distributions are rather identical for both the SSPD and the ionization detector. They are both consistent with the arrival of individual molecules. The absence of any signal related to the individual carrier gas atoms is a hint to an energy threshold in the SSPD which can only be overcome by sufficiently massive and energetic molecules. In our experiments the least energetic molecules detected by the SSPD were tryptophan particles in the velocity band of $300 . .500 \mathrm{~m} / \mathrm{s}$, i.e. with a kinetic energy of $100 . .300 \mathrm{meV}$ if we assume to see isolated molecules.

Compared to that value, all rare gas beams have still too little kinetic energy to be detected. Helium at $800 \mathrm{~m} / \mathrm{s}$ reaches only $10 \mathrm{meV}$ and xenon at $250 \mathrm{~m} / \mathrm{s}$ would only attain $40 \mathrm{meV}$ - still well below the value for tryptophan, which carries also internal energy in addition.

\section{CONCLUSION}

The present experiments are just a promising start of an interesting journey into single neutral molecule detection using superconducting single photon detectors. Our experiments give good first evidence that SSPDs can be used to register the incidence of neutral single nanoparticles.

As of today there is no efficient easy-to-implement way of detecting neutral large proteins to cross-check our results with individual, isolated insulin, hemoglobin or myoglobin. But we see a good consistence between the SSPD results and photoionization mass spectrometry in the flight times for tryptophan and gramicidin.

One might furthermore ask whether a high internal excitation may also add sufficient energy to the chip, which will be tested in future experiments by systematically varying the internal temperature of the molecules.

The possibility of detecting more massive neutral and labile molecules is promising for many applications in physical chemistry and also for matter wave interferometry. Even if the SSPD method, cannot (yet) discriminate between different masses, de Broglie interferometry itself has been shown to be capable of discriminating different molecular properties [5] and experiments with clean and mass selected sources would only require a good sensitivity to the existence, not to the mass of the particle.

The technology certainly requires further development and exploration but it may allow us to close a 'detector loophole' for particles which are too complex to be efficiently photoionized and yet too small to be well detected by other means.

\section{ACKNOWLEDGMENTS}

This work was supported by the Austrian Science Funds FWF within the Wittgenstein program Z149 as well as by the Italian Fondazione Angelo Della Riccia. We thank Sanofi-Aventis Inc. for the donation of pure insulin powder. This work was also supported by the Russian Agency of Science and Innovation (contract 02.513.11.3446) and the Russian Foundation for Basic Research (grant 09-02-12364).

\section{REFERENCES}


[1] Christopher A Noble and Kimberly A Prather. Real-time single particle mass spectrometry: A historical review of a quarter century of the chemical analysis of aerosol. Mass Spectrom. Rev., 19:248-274, 2000.

[2] W. Schöllkopf and J.P. Toennies. The nondestructive detection of the helium dimer and trimer. J. Chem. Phys., 104:1155-1158, 1996.

[3] M. Arndt, O. Nairz, J. Voss-Andreae, C. Keller, G. Van der Zouw and A. Zeilinger . Wave-particle duality of C60 molecules. Nature, 401:680-682, 1999.

[4] L. Hackermüller, S. Uttenthaler, K. Hornberger, E. Reiger, B.Brezgerand, A. Zeilinger and M. Arndt. Wave nature of biomolecules and fluorofullerenes. Phys. Rev. Lett., 91:90408, 2003.

[5] Stefan Gerlich, Michael Gring, Hendrik Ulbricht, Klaus Hornberger, Jens Tüxen, Marcel Mayor and Markus Arndt. Matter-wave metrology as a complementary tool for mass spectrometry. Angew. Chem. Int. Ed. Engl., 47(33):6195-8, 2008.

[6] Hendrik Ulbricht, Martin Berninger, Sarayut Deachapunya, Andre Stefanov and Markus Arndt Gas phase sorting of fullerenes, polypeptides and carbon nanotubes. Nanotechnology, 19:045502, 2008.

[7] E. W. Schlag and J. Grotemeyer. Do large molecules ionize? Chem. Phys. Lett., 190:521-527, 1992.

[8] C. H. Becker and K. J. Wu. On the photoionization of large molecules. J. Am. Soc. Mass Spectrom., 6:883888, 1995.

[9] M. Marksteiner, P. Haslinger, M. Sclafani, H. Ulbricht and M. Arndt. Gas-Phase Formation of Large Neutral Alkaline-Earth Metal Tryptophan Complexes. J. Am. Soc. Mass Spectr., 19(7):1021-1026, 2008.

[10] M. Marksteiner, P. Haslinger, M. Sclafani,H. Ulbricht and M. Arndt. UV and VUV ionization of organic molecules, clusters and complexes. J. Phys. Chem. A, DOI: 10.1021/jp905039f, 2009.

[11] Elisabeth Reiger, Sander Dorenbos, Valery Zwiller, Alexander Korneev, Galina Chulkova, Irina Milostnaya, Olga Minaeva, Gregory Goltsman, Jennifer Kitaygorsky, Dong Pan, Wojtek Slysz, Arturas Jukna and Roman Sobolewski. Spectroscopy with nanostructured superconducting single photon detectors. IEEE J. Sel. Top. Quant. Electr., 13:934-943, 2007.

[12] Aviv Amirav. Electron impact and hyperthermal surface ionization mass spectrometry insupersonicmolecular beams. Org. Mass Spectr., 26:1-17, 1991.

[13] C. Weickhardt, L. Draack and A. Amirav. Laser desorption combined with hyperthermal surface ionization time-of-flightmassspectrometry. Anal. Chem., 75:5602, 2003.

[14] B. Ilic, H. G. Craighead, S. Krylov, W. Senaratne, C. Ober and P. Neuzil. Attogram detection using nanoelectromechanical oscillators. J. Appl. Phys., 95:36943703, 2004.

[15] Mo Li, H. X. Tang and M. L. Roukes. Ultra-sensitive nems-based cantilevers for sensing, scanned probe and very high-frequency applications. Nature Nanotech., $2: 114-120,2007$.

[16] K. Jensen, K. Kim and A. Zettl. An atomicresolution nanomechanical mass sensor. Nature Nanotech., 3:533537, 2008.
[17] A. K. Naik, M. S. Hanay, W. K. Hiebert, X. L. Feng and M. L. Roukes. Towards single-molecule nanomechanical mass spectrometry. Nature Nanotech., Advanced online: 21 June 2009:1-6, 2009.

[18] Frank J. Low. Low-temperature germanium bolometer. J. Opt. Soc. Am., 51:1300-1304, 1961.

[19] H Kraus. Superconductive bolometers and calorimeters. Supercond. Sci. Technol., 9:827842, 1996.

[20] C. Enss and D. McCammon. Physical principles of low temperature detectors: Ultimate performance limits and current detector capabilities. J. Low. Temp. Phys., 151:524, 2008.

[21] M. Cavallini, L. Meneghetti, G. Scoles and M. Yealland. Molecular beam scattering apparatus with low temperature bolometer detector. Rev. Sci. Instr., 42:1759, 1971.

[22] DH Andrews, RM Milton and W Desorbo. A fast superconducting bolometer. J. Opt. Soc. Am., 36:518-524, 1946.

[23] Norman E Booth and David J Goldie. Superconducting particle detectors. Supercond. Sci. Technol., 9:493-516, 1996.

[24] M. Frank, C. A. Mean, Simon E. Labov, W. H. Benner, D. Horn, J. M. Jaklevic and A. T. Barfknecht. Highefficiency detection of 66000 da protein molecules using a cryogenic detector in a matrix-assisted laser desorption/ionization time-of-flight mass spectrometer. Rap. Comm. Mass Spectr., 10:1946-1950, 1996.

[25] Damian Twerenbold, Jean-Luc Vuilleumier, Daniel Gerber, Almut Tadsen, Ben van den Brandt and Patrick M. Gillevet. Detection of single macromolecules using a cryogenic particle detector coupled to a biopolymer mass spectrometer. Appl. Phys. Lett., 68:3503-3505, 1996.

[26] E. Esposito, R. Cristiano, S. Pagano, D. Perez de Lara and D. Twerenbold. Fast josephson cryodetector for time of flight mass spectrometry. Physica C, 372-376:423 - 426, 2002.

[27] Damian Twerenbold, Daniel Gerber, Dominique Gritti, Yvan Gonin, Alexandre Netuschill, Frédéric Rossel, Dominique Schenker and Jean-Luc Vuilleumier. Single molecule detector for mass spectrometry with mass independent detection efficiency. Proteomics, 1:66-69, 2001.

[28] G. N. Goltsman, O. Okunev, G. Chulkova, A. Lipatov, A. Semenov, K. Smirnov, B. Voronov, A. Dzardanov, C. Williams and Roman Sobolewski. Picosecond superconducting single-photon optical detector. Appl. Phys. Lett., 79:705-707, 2001.

[29] Alex D. Semenov, Gregory N. Goltsman and Alexander A. Korneev. Quantum detection by current carrying superconducting film. Physica C, 351:349-356, 2001.

[30] A. Verevkin, J. Zhang, Roman Sobolewski, A. Lipatov, O. Okunev, G. Chulkova, A. Korneev, K. Smirnov, , G. N. Goltsman and A. Semenov. Detection efficiency of large-active-area nbn single-photon superconducting detectors in the ultraviolet to near-infrared range. Appl. Phys. Lett., 80:4687-4689, 2002.

[31] A. Korneev, V. Matvienko, O. Minaeva, I. Milostnaya, I. Rubtsova, G. Chulkova, K. Smirnov, V. Voronov, G. Goltsman, W. Slysz, A. Pearlman, A. Verevkin and Roman Sobolewski. Quantum efficiency and noise equivalent power of nanostructured, nbn, single-photon detectors in the wavelength range from visible to infrared. 
IEEE Transact. Appl. Supercond., 15:571-574, 2005.

[32] G. Goltsman, O. Minaeva, A. Korneev, M. Tarkhov, I. Rubtsova, A. Divochiy, I. Milostnaya, G. Chulkova, N. Kaurova, B. Voronov, D. Pan, A. Cross, A. Pearlman, I. Komissarov, W. Slysz and R. Sobolewski. Middleinfrared to visible-light ultrafast superconducting singlephoton detector. IEEE Transact. Appl. Supercond., $17(1): 246-251,2007$.

[33] G. Gol'tsman, K. Smirnov, P. Kouminov, B. Voronov, N. Kaurova, V. Drakinsky, J. Zhang, A. Verevkin and
R. Sobolewski. Fabrication of nanostructured superconducting single-photon detectors. IEEE Transact. Appl. Supercond., 13(2):192-195, June 2003.

[34] Claus Köster and Jürgen Grotemeyer. Single-photon and multi-photon ionization of infrared laser-desorbed biomolecules. Org, Mass Spectr., 27:463-471, 1992.

[35] Jh. Arps, Ch. Chen, Mp. McCann and I. Datskou. Ionization of organic-molecules using coherent vacuum ultraviolet-light. Appl. Spectr., 43(7):1211-1214, 1989. 